\title{
Aboriginal Australian
}

National Cancer Institute

\section{Source}

National Cancer Institute. Aboriginal Australian. NCI Thesaurus. Code C154875.

The native people of Australia. 